05,08,13

\title{
Вихреподобные образования на дефектах магнитоодноосных пленок
}

\author{
(C) Р.М. Вахитов ${ }^{1}$, А.А. Ахметова ${ }^{1}$, Р.В. Солонецкий ${ }^{2}$ \\ ${ }^{1}$ Физико-технический институт, Башкирский государственный университет, \\ Уфра, Россия \\ 2 Уфимский государственный авиационный технический университет, \\ Уфра, Россия \\ E-mail: VakhitovRM@Yahoo.com
}

(Поступила в Редакцию 4 сентября 2018 г. В окончательной редакции 5 октября 2018 г.)

\begin{abstract}
Теоретически исследуются магнитные неоднородности, образующиеся на колумнарных дефектах типа потенциальная яма в магнитоодноосных пленках. Показано, что при определенных условиях на таких дефектах возникают вихреподобные образования с распределением намагниченности, имеющим три участка вращения магнитных моментов. Из анализа структуры и свойств вихреподобных неоднородностей в зависимости от материальных параметров следует, что они в основном определяются размерами дефектов и глубиной потенциальной ямы. Приведены экспериментальные данные, касающиеся их существования.
\end{abstract}

DOI: 10.21883/FTT.2019.03.47235.248

\section{1. Введение}

Среди различных видов дефектов, присутствующих в магнитных материалах, особый интерес представляют дефекты типа „потенциальная яма““ [1]. Они характерны тем, что их наличие вносит отрицательный вклад в энергию магнетика, что может привести к образованию на них магнитных неоднородностей различной топологии [2-5]. С ними связан один из основных механизмов возникновения гистерезисных явлений в материалах при их перемагничивании, который проявляется в виде задержки и роста зародышей перемагничивания $[2,6]$. Из расчетов следует $[1,5]$, что в одноосных пленках на дефектах такого рода могут возникнуть два типа магнитных образований, соответствующих $0^{0}$ доменным границам (ДГ) и различающихся энергией, шириной и амплитудой (максимальным углом отклонения $\theta_{m}$ вектора намагниченности М от равновесного направления $\mathbf{M}_{0}$ в доменах). Отличия в их структуре существенно сказываются на свойствах этих неоднородностей и позволяют их идентифицировать при экспериментальном исследовании магнитных образований на дефектах пленок. В частности, если их наблюдать в поляризационном микроскопе с помощью магнитооптических методов, то высокоамплитудные неоднородности $\left(\pi<\theta_{m}<3 \pi / 2\right)$ из-за наличия в их структуре области, в которой магнитные моменты противоположно направлены намагниченности $\mathbf{M}_{0}$, будут отличаться от малоамплитудной неоднородности $\left(\theta_{m}<\pi / 2\right)$ наличием ореола [7]. Такую картину действительно можно наблюдать на фотографиях, полученных при сканировании поверхности феррит-гранатовой пленки с последующей фотосъемкой [5]. Однако в реальных магнетиках имеющиеся в них дефекты являются, по крайней мере, двумерными объектами. Следовательно, результаты, полученные в рамках одномерной теории [1], могут дать не совсем адекватное описание магнитных образований на двумерных дефектах. Из предварительных расчетов следует [8], что в магнитоодноосных пленках на колумнарных дефектах [9] типа „потенциальная яма“ при определенных значениях параметров материала могут существовать вихреподобные магнитные неоднородности.

Известно, что вихревые состояния в магнетиках, являющиеся разновидностью 2D-топологических солитонов, теоретически были предсказаны задолго до их экспериментального обнаружения. Аналитические решения, описывающие структуру магнитного вихря, были впервые предложены в работе [10]. Они послужили отправной точкой для дальнейших исследований в этой области, среди которых можно отметить [11-14]. Однако после экспериментального обнаружения магнитных вихрей в пермаллоевых наноточках [15] интерес к ним еще сильнее возрос [16], что было связано с открывшимися перспективами их применения в устройствах магнитной памяти с высокой плотностью записи информации и произвольного доступа (MRAM). Кроме того, магнитные вихри привлекают внимание своей необычной структурой и наличием нетривиальных статических и динамических свойств [16]. В связи со сказанным, представляет также интерес изучение вихреподобных магнитных состояний, образующихся на колумнарных дефектах типа потенциальная яма, в одноосных ферромагнитных пленках.

Необходимо отметить, что исследования по изучению взаимодействия магнитных вихрей с дефектами, присутствующими в магнитных наноточках, проводились и не однократно [17-20], так как они влияют на свойства данных вихрей: на их траекторию, на размеры и т.д. Однако, в предлагаемой постановке задачи, в которой наличие дефектов в магнетике является условием существования магнитных вихрей, в работах [17-20] не рассматривались. Кроме того, дополнительным стимулом 
для проведения данных исследований может послужить тот факт, что подобные дефекты можно создавать целенаправленно, например, с помощью сфокусированного лазерного облучения участков поверхности магнитной пленки, меняющего значения его параметров. В частности, в работе [21] в местах локального воздействия было обнаружено зарождение вертикальных блоховских линий, а в [22] в облученных участках пленки наблюдались кольцевые структуры.

\section{2. Магнитные образования на колумнарном дефекте. Основные уравнения}

Рассмотрим неограниченную одноосную ферромагнитную пленку (толщиной $D$ ), содержащую дефект в форме сквозного цилиндра (колумнарный дефект [9]). Учитывая симметрию магнетика, выберем цилиндрическую систему координат $(r, \varphi, z)$ с центром $O$, совпадающим с центром окружности колумнарного дефекта и расположенным в середине (по толщине) пленки. Соответственно, материальные параметры образца $(A-$ обменный параметр, $K_{u}$ - константа одноосной анизотропии, $M_{s}$ - намагниченность насыщения) и единичный вектор намагниченности $\mathbf{m}=\mathbf{M} / M_{s}$ зависят только от переменной $r$. Нетрудно показать, что магнитные неоднородности, имеющие место в таком магнетике, имеют блоховскую структуру [23] и, следовательно, вектор $\mathbf{m}$ будет описываться только одним углом $\theta$, отчитывающимся от оси $O z$. Однако такая ситуация может измениться в ультратонких пленках, когда $D \ll \Delta_{0}$ [24], где $\Delta_{0}-$ характерный размер ДГ в одноосных ферромагнетиках [25]. В силу сказанного будем считать, что $D \gg \Delta_{0}$.

Тогда энергия магнитных неоднородностей рассматриваемого магнетика с учетом обменного взаимодействия (характеризуемого обменным параметром $A$ ), одноосной анизотропии $\left(K_{u}\right)$ и размагничивающих полей, обусловленных конечностью пленки, запишется в виде:

$$
\begin{aligned}
& E=2 \pi D \int_{0}^{\propto}\left\{A\left[\left(\frac{d \theta}{d r}\right)^{2}+\frac{\sin ^{2} \theta}{r^{2}}\right]+K_{u} \sin ^{2} \theta\right. \\
& +\frac{2 M_{s} \cos \theta}{D} \int_{0}^{\propto}\left\{M_{s}^{\prime} \cos \theta^{\prime}\left[k_{0} \mathrm{~K}\left(k_{0}\right)-k \mathrm{~K}(k)\right] \sqrt{\frac{r^{\prime}}{r}} d r^{\prime}\right\} r d r
\end{aligned}
$$

где

$$
\begin{gathered}
k_{0}=\frac{2 \sqrt{r r^{\prime}}}{r+r^{\prime}}, \quad k=\frac{2 \sqrt{r r^{\prime}}}{\sqrt{\left(r+r^{\prime}\right)^{2}+D^{2}}}, \\
K(s)=\int_{0}^{\pi / 2} \frac{d \varphi}{\sqrt{1-s^{2} \sin ^{2} \varphi}}
\end{gathered}
$$

- полный эллиптический интеграл І-го рода, $s-$ его модуль, $M_{s}^{\prime}=M_{s}\left(r^{\prime}\right), \theta^{\prime}=\theta\left(r^{\prime}\right)$.
В качестве модели дефекта рассмотрим структурную неоднородность магнетика, материальные параметры $P=\left\{A, K_{u}, M_{s}\right\}$ которой изменяются скачком в области дефекта $[1,26]$ следующим образом

$$
P= \begin{cases}P_{1}, & r \geq R_{0} \\ P_{2}, & r \leq R_{0}\end{cases}
$$

где $P_{i}=\left\{A_{i}, K_{u i}, M_{s i}\right\}$ - материальные параметры вне колумнарного дефекта $(i=1)$ и в области дефекта $(i=2)$.

Соответствующее уравнение Эйлера-Лагранжа, описывающее распределение намагниченности в области дефекта, примет вид

$$
\begin{aligned}
& \frac{1}{r} \frac{d}{d r}\left(A r \frac{d \theta}{d r}\right)-\sin \theta \cos \theta\left(K_{u}+\frac{A}{r^{2}}\right)+\frac{2 M_{s}}{D^{2}} \\
& \quad \times \sin \theta \int_{0}^{\infty} M_{s}^{\prime} \cos \theta^{\prime}\left[k_{0} \mathrm{~K}\left(k_{0}\right)-k \mathrm{~K}(k)\right] \sqrt{\frac{r^{\prime}}{r}} d r^{\prime}=0 .
\end{aligned}
$$

Особенностью соотношения (1) для энергии $E$, а также уравнения (3), является наличие в выражении для обменной энергии второго слагаемого, которое по характеру зависимости от угла $\theta$ можно отнести к одноосной анизотропии. Это вполне логично, так как обменное взаимодействие заставляет соседние спины быть направленными коллинеарно друг другу, что приводит к уширению ДГ. Однако если микромагнитная структура, образующаяся в пленке, обладает цилиндрической симметрией, то ДГ по форме является уже круговой. В этом случае обменное взаимодействие для части магнитных моментов, находящихся внутри цилиндрической области, ограниченной ДГ, приводит к их обменному „сжатию“, что выражается в наличии вышеуказанного слагаемого, которое вносит положительный вклад в одноосную анизотропию. Наличие такого вклада обуславливает при определенных условиях возможность существования магнитных вихрей $[10,16]$, а также цилиндрических магнитных доменов [27]. Кроме того, как известно [27], условием существования последних в одноосных ферромагнитных пленках считается выполнение неравенства $Q>1\left(Q=K_{u} / 2 \pi M_{s}^{2}\right.$ - фактор качества), так как при $Q<1$ размагничивающие поля заставляют спины ложиться в плоскость пленки. Однако в работе [28] было показано, что существование таких доменов возможно и в пленках с $Q<1$ (вплоть до значений $Q \approx 0,75)$, что связано с вкладом обменного взаимодействия в одноосную анизотропию. Это исследование (ранее не получившее адекватного объяснения) как раз говорит о важной роли, которую играет данное слагаемое в теории микромагнитных структур, имеющих аксиальную симметрию. 


\section{3. Особенности структуры вихреподобной неоднородности, образующейся на колумнарных дефектах}

Численный анализ интегро-дифференциального уравнения (6) с учетом (4), проведенный методом, предложенным в [5] и апробированным в [29], позволяет найти распределение намагниченности $\mathbf{m}$ в области колумнарного дефекта, и его характеристики: характерные размеры магнитной неоднородности и ее энергию. При этом необходимо отметить, что все параметры, имеющие размерность длины, приведены к величине $\Delta_{1}=\sqrt{A_{1} / K_{u 1}}$ (здесь $\Delta_{1}=\Delta_{0}$ ). Из расчетов следует, что эта неоднородность на дефекте представляет вихреподобное образование (рис. 1) с ядром (кор) в центре $\left(m_{z}(0)=-1\right)$ и с пологим участком вращения магнитных моментов в области дефекта, в котором происходит задержка их вращения вблизи плоскости, параллельной поверхности пленки $\left(z= \pm D / 2, r<R_{0}\right)$. Вне области дефекта $\left(r>R_{0}\right)$ на графике зависимости $m_{z}=m_{z}(r)$ имеет место резкий подъем, соответствующий тому, что магнитные моменты на этом участке наискорейшим образом стремятся сориентироваться вдоль оси $O z$ $\left(m_{z}=1\right)$. В результате в структуре вихреподобного магнитного образования можно выделить три характерных участка вращения магнитных моментов: центральное ядро (кор), в котором основную роль играют одноосная анизотропия обменного происхождения и магнитостатические поля, пологий (средний) участок, где доминирует легкоплоскостная анизотропия дефекта, и участок, расположенный на границе дефекта, где происходит ускоренное вращение магнитных моментов из-за преобладающего влияния одноосной анизотропии типа легкая ось. Соответственно, на графике (рис. 1) имеются

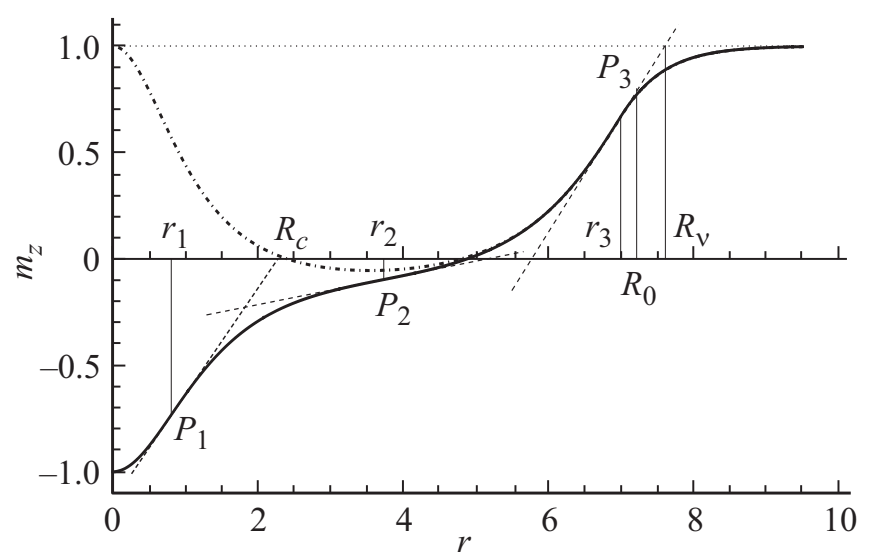

Рис. 1. Распределение намагниченности в вихреподобном образовании с полярностью $m_{z}(0)=1$ (штрихпунктирная линия) и $m_{z}(0)=-1$ (сплошная линия) при следующих значениях материальных параметров: $K_{u 2}=-K_{u 1}, R_{0}=7, A_{2}=A_{1}, Q=5$, $M_{s 2}=M_{s 1}, D=10$. Здесь штриховыми линиями обозначены касательные к соответствующим точкам перегиба.

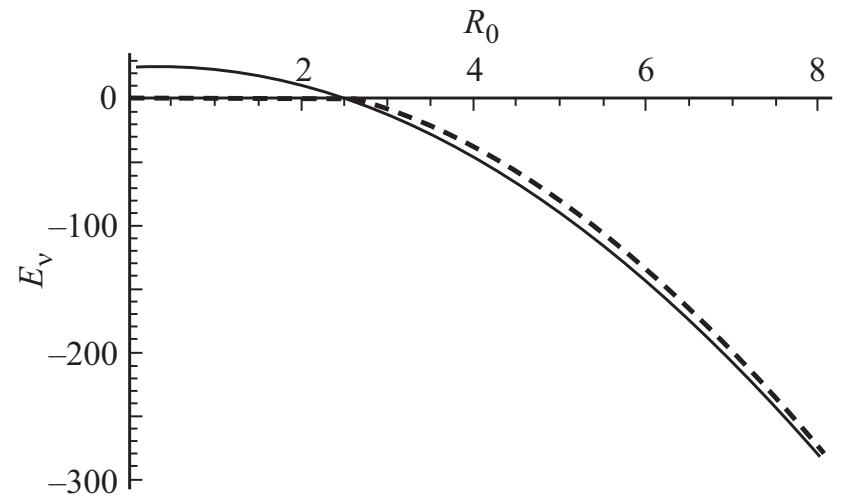

Рис. 2. График зависимости энергии вихреподобного образования с полярностью $m_{z}(0)=-1$ (сплошная линия) и $m_{z}(0)=1$ (штриховая линия) от радиуса дефекта при следующих значениях материальных параметров: $K_{u 2}=-K_{u 1}$, $A_{2}=A_{1}, Q=2, M_{s 2}=M_{s 1}, D=10$.

три точки перегиба $\left(P_{1}, P_{2}, P_{3}\right)$, из которых крайняя справа $\left(P_{3}\right)$, соответствующая большему значению $r$ и расположенная в граничном участке, будет определять размер $R_{v}$ вихреподобного состояния согласно соотношению [30]

$$
R_{v}=r_{3}+\frac{1-m_{z}\left(r_{3}\right)}{m_{z}^{\prime}\left(r_{3}\right)}
$$

где $r_{3}-$ есть радиальная координата точки $P_{3}$, $m_{z}^{\prime}\left(r_{3}\right)$ - значение производной функции $m_{z}(r)$ по переменной $r$ при $r=r_{3}$. В данном случае $R_{v}$ определяется как координата точки пересечения касательной, проведенной через крайнюю справа точку перегиба $r_{3}$, с прямой $m_{z}(r)=1$. Такое магнитное образование с тремя точками перегиба в литературе иногда называют „перетяжкой“ [31]. В магнетиках с доменной структурой она играет роль зародыша новой фазы при спин-переориентационном фазовом переходе I рода. В рассматриваемом случае перетяжка является характерной чертой структуры вихреподобной неоднородности.

Следует отметить, что возможно существование еще одного типа магнитной неоднородности, представляющей также вихреподобное образование на дефекте, но с другой полярностью кора $\left(m_{z}(0)=1\right.$, (рис. 1$\left.)\right)$. Они различаются энергетически (рис. 2): неоднородность первого типа $\left(m_{z}(0)=-1\right)$ является энергетически более выгодной, чем неоднородность второго типа $\left(m_{z}(0)=1\right)$. При этом в точке пересечения графиков их энергий, которая находится на оси $O-R_{0}$, неоднородность с полярностью $\left(m_{z}(0)=1\right)$ исчезает и ее энергия зануляется. Разность их энергий $\Delta E$ определяется величиной их магнитостатической энергии, заключенной в области, занимаемой кором. Поэтому она зависит как от радиуса дефекта $R_{0}$, так и от фактора качества $Q:$ с возрастанием $Q$ эта разность быстро уменьшается и при $Q>10$ асимптотически стремится к некоторому предельному 


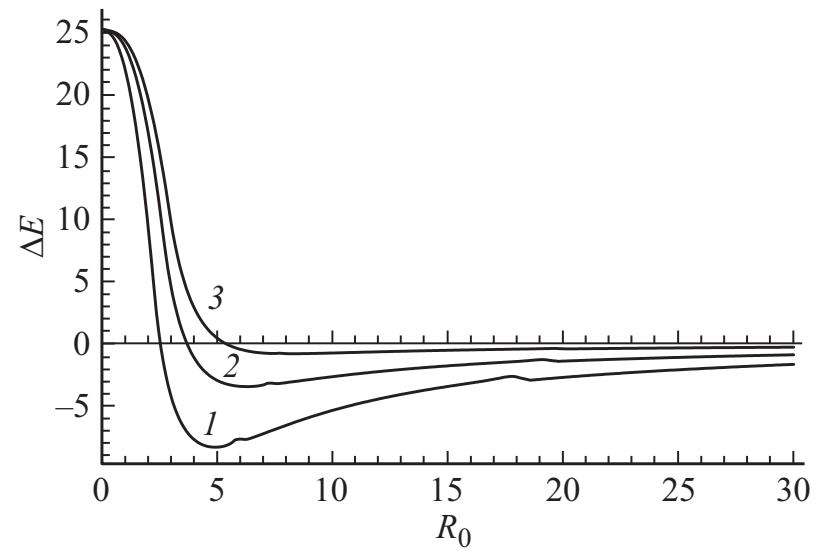

Рис. 3. График зависимости разности энергий вихреподобных образований с полярностью $m_{z}(0)=1$ и $m_{z}(0)=-1$ от радиуса дефекта. Значения материальных параметров (за исключением $Q$ ) те же, что и на рис. 2. Линия 1 соответствует $Q=2$, линия $2-Q=5$, линия $3-Q=20$.

значению (рис. 3). В то же время при увеличении $R_{0}$, разность $\Delta E$ увеличивается, хотя и незначительно.

Размер кора $R_{C}$ можно определить как координату точки пересечения касательной, проведенной через точку перегиба $P_{1}$ графика функции $m_{z}=m_{z}(r)$ с прямой $m_{z}=0$ (рис. 1). В данном случае величина $R_{C}$ находится по той же формуле [30], что и соотношение (4)

$$
R_{C}=r_{1}-\frac{m_{z}\left(r_{1}\right)}{m_{z}^{\prime}\left(r_{1}\right)}
$$

Прежде всего, отметим, что не при всех значениях параметров материала и характеристик дефекта возможно существование трех точек перегиба и тем самым образование вихреподобной микромагнитной структуры с тремя участками вращения магнитных моментов, о которых говорилось выше. В частности, из расчетов следует, что при небольших значениях параметров $R_{0}$ и $\left|K_{u 2}\right|$ в структуре магнитного образования на дефекте будет одна точка перегиба, то есть перетяжка отсутствует. В данном случае энергия вихреподобного образования лежит в положительной области ее значений, что говорит о невозможности его существования на колумнарном дефекте в рассматриваемых пленках.

Согласно расчетам (рис. 4) радиус кора $R_{C}$ вихреподобного образования с возрастанием размера дефекта $R_{0}$ резко увеличивается вплоть до $R_{0} \sim 7 \Delta_{1}$, а затем асимптотически стремится к некоторому предельному значению $R_{\text {пр }}$, величина которого меняется в зависимости от глубины потенциальной ямы $K_{u 2}$ и фактора качества $Q$. Очевидно, величина $R_{\text {пр }}$ определяется конкуренцией обменного взаимодействия, размагничивающих полей кора и поля, создаваемого легкоплоскостной анизотропией колумнарного дефекта, причем первый и третий фактор оказывает существенное влияние при малых значениях радиальной переменной, а второй и третий факторы при больших $r$.
Зависимость размера кора от глубины потенциальной ямы $K_{u 2}$ ведет несколько иначе: с увеличением $\left|K_{u 2}\right|$ paдиус кора уменьшается: сначала резко, а потом асимптотически приближается к своему предельному значению. Такая зависимость объясняется тем, что вращательные моменты легкоплоскостной анизотропии стремятся повернуть магнитные моменты в сторону плоскости пленки: чем больше глубина потенциальной ямы, тем легче они ложатся в плоскость. В этом случае касательная к графику зависимости $m_{z}=m_{z}(r)$ пологого участка вращения магнитных моментов (рис. 1) стремится быть параллельной плоскости $z=0$ (в пределе).

Размер вихреподобного образования определяется, прежде всего, размером дефекта $R_{0}$ и глубиной потенциальной ямы $K_{u 2}$. Как видно из графиков зависимостей $R_{v}$ от $R_{0}$ (рис. 5), при определенном значении размера дефекта имеется скачкообразный характер этой зависимости, который связан с изменением топологии

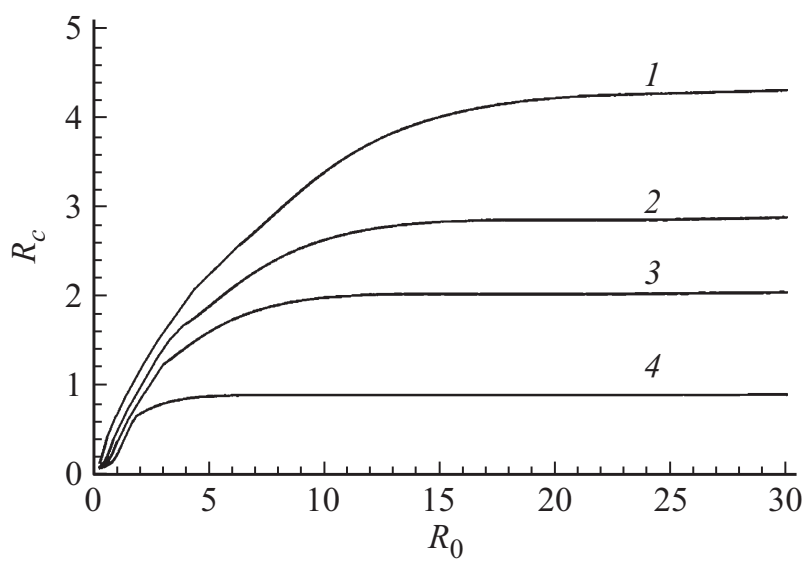

Рис. 4. График зависимости размера кора вихреподобного образования с полярностью $m_{z}(0)=-1$ от радиуса дефекта при следующих значениях материальных параметров: $K_{u 2}=-K_{u 1}$, $A_{2}=A_{1}, Q=5, M_{s 2}=M_{s 1}, D=10$. Здесь линия 1 соответствует $K_{u 2}=-0.2 K_{u 1}, 2-K_{u 2}=-0.5 K_{u 1}, 3-K_{u 2}=-K_{u 1}$, $4-K_{u 2}=-5 K_{u 1}$.

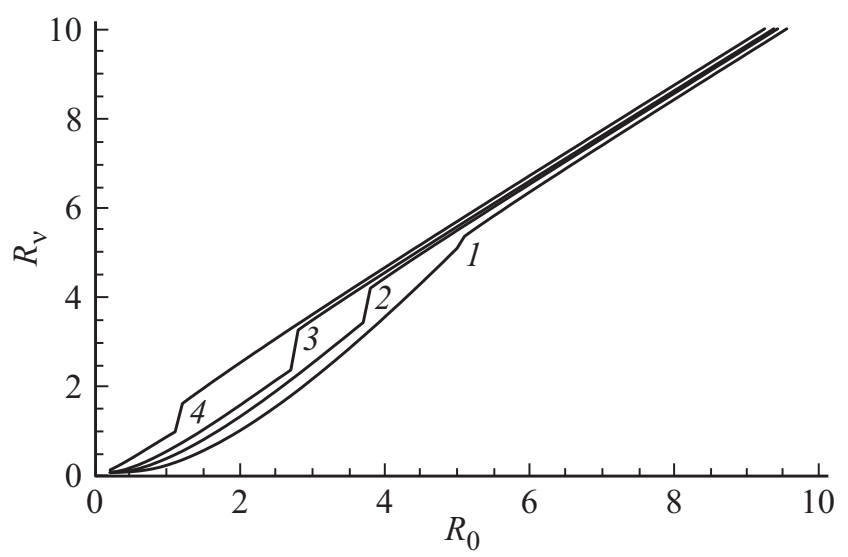

Рис. 5. График зависимости размера вихреподобного образования от радиуса дефекта. Значения материальных параметров, обозначения кривых и полярность кора те же, что и на рис. 4. 
вихреподобной неоднородности и образованием на ней перетяжки. Значение радиуса, при котором происходит такой переход, существенно зависит от величины $K_{u 2}$ : чем больше глубина потенциальной ямы, тем при меньших размерах дефекта такой переход имеет место. Поскольку энергия магнитной неоднородности до перехода положительна, то условия для ее существования отсутствуют. В этом случае найденные предельные значения радиуса перехода могут служить нижней границей области существования вихреподобного состояния $R_{0}$. При дальнейшем возрастании $R_{0}$, величина $R_{v}$ также увеличивается, становясь линейной функцией от $R_{0}$. Это согласуется с эмпирически установленной закономерностью $[1,4,5,26]$, утверждающей, что магнитная неоднородность, образующаяся на дефекте, подстраивается под его профиль.

\section{4. Диаграммы устойчивых состояний вихреподобных образований}

Очевидно, на устойчивые состояния вихреподобных неоднородностей будет оказывать влияние также размагничивающие поля пленки, вклад которых в энергию магнетика (1) обратно пропорционален фактору качества $Q$. Анализ его влияния, как и в одномерном случае, рассмотрим на диаграмме устойчивых состояний вихреподобных неоднородностей в переменных $Q-R_{0}$, которая представлена на рис. 6. Из нее видно, что область устойчивости магнитной неоднородности с полярностью $m_{z}(0)=-1$ по параметру $Q$ ограничена снизу некоторой кривой $Q=Q_{1}\left(R_{0}\right)$, для которой максимальная величина $Q$ достигает некоторого значения $Q_{\max }$ (при данных параметрах материала $\left.Q_{\max }=1.4\right)$, а минимальное значение $Q_{\min } \rightarrow 1$ при больших значениях $R_{0}$.

Эта кривая, являющаяся нижней границей области устойчивости вихреподобных неоднородностей, существенно зависит от глубины потенциальной ямы $K_{u 2}$ : чем больше $\left|K_{u 2}\right|$, тем она ближе к критической линии $Q_{0}=1$ и наоборот. Найденная закономерность обусловлена характером влияния величины $K_{u 2}$ на устойчивые состояния вихреподобных образований: при возрастании $\left|K_{u 2}\right|$, уменьшается энергия дефекта. Соответственно возрастают вращательные моменты, обусловленные легкоплоскостной анизотропией и удерживающие вихреподобное образование на дефекте, и тем самым его устойчивость относительно внешних возмущений возрастает.

Кривая $Q=Q_{1}\left(R_{0}\right)$ практически не зависит от $M_{s 2}$, а если есть такая зависимость, то при малых $D$, точнее при $D \ll \Delta_{1}$ [24]. Однако, в этом случае модель уже не описывает вихреподобную неоднородность, образующуюся на колумнарном дефекте.

В то же время, как показывают расчеты, граница области устойчивости зависит от параметра $A_{2}$ : при его возрастании (по сравнению с $A_{1}$ ) кривая $Q=Q_{1}\left(R_{0}\right)$ смещается вверх и, наоборот, при понижении $A_{2}$, она смещается вниз. Такое поведение кривой в зависимости

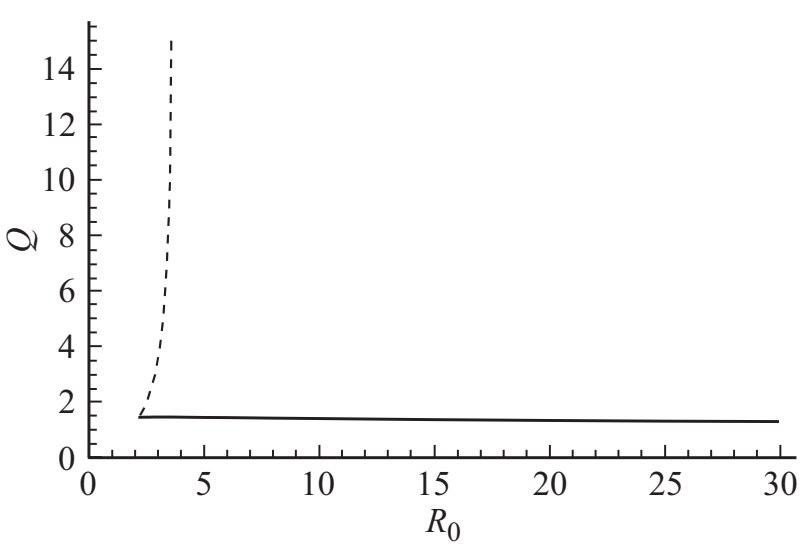

Рис. 6. Диаграмма области устойчивости вихреподобного образования с полярностью $m_{z}(0)=-1$. Здесь $K_{u 2}=-K_{u 1}$, $A_{2}=A_{1}, M_{s 2}=M_{s 1}, D=10$.

от $A_{2}$ объясняется тем, что обменное взаимодействие вносит положительный вклад в энергию (1) и при увеличении $A_{2}$ этот вклад возрастает, что сказывается на области устойчивости вихреподобных образований.

Слева область устойчивости ограничена другой кривой $Q=Q_{2}\left(R_{0}\right)$, которая также зависит от $K_{u 2}$ и $M_{s 2}$, в частности, при возрастании $\left|K_{u 2}\right|$ эта кривая смещается влево поближе к оси ординат $Q$ и, следовательно, область устойчивости вихреподобных образований расширяется в полном соответствии с ранее сказанным. Подобным же образом ведет эта кривая при возрастании параметра $M_{s 2}$. В этом случае размагничивающие поля вихреподобного образования с полярностью кора $m_{z}(0)=-1$ вносят отрицательный вклад в энергию магнетика. В частности, для однородного состояния магнетика их вклад в энергию (1) приводит к перенормировке константы одноосной анизотропии: $K_{u}^{\prime}=K_{u}-2 \pi M_{s}^{2}$. Учитывая, что в области дефекта $K_{u}=K_{u 2}<0$, то при возрастании параметра $M_{s 2}$ увеличивается глубина потенциальной ямы. При наличии доменной структуры этот вклад в энергию также отрицателен, однако в выражении для $K_{u}^{\prime}$ коэффицент во втором члене будет уже другим [27].

\section{5. Обсуждение результатов}

Из приведенных расчетов структуры и устойчивости вихреподобных образований следует, что они могут возникать в магнитоодноосных пленках на колумнарных дефектах типа потенциальная яма. Топология и размеры этих неоднородностей существенно зависят от характеристик дефекта $\left(A_{2}, K_{u 2}, M_{s 2}, R_{0}\right)$ и прежде всего от его радиуса и глубины потенциальной ямы. По структуре они представляют магнитную неоднородность, подобную магнитному вихрю, но имеющую более сложное распределение вектора $\mathbf{m}$, содержащее три характерных участка вращений магнитных моментов: кор, пологий 
(средний) и граничный участки. Микромагнитную структуру, подобную описанной выше неоднородности, предположительно наблюдали в работе [5]. Следует отметить также работу [32], в которой вполне обоснованно утверждается о наблюдении вихреподобных образований на дефектах типа потенциальная яма, создаваемых искусственно. В данной работе исследовались многослойные пленки $\mathrm{Co} / \mathrm{Pt}$ с перпендикулярной анизотропией, предварительно облученные сфокусированным пучком ионов $\mathrm{He}^{+}$локальных участков поверхности образца. В этом случае в участках, подвергшихся облучению, будет иметь место пониженное значение константы перпендикулярной анизотропии, характер которой зависит от дозы облучения [33]. Таким способом в многослойной пленке [32] была сформирована регулярная структура колумнарных дефектов, представляющих потенциальную яму для вихреподобных образований. Изображения последних, полученные методом магнито-силовой микроскопии, качественно совпадают с вихреподобными структурами, изученными здесь. К сожалению, их количественное сравнение не представляется возможным, так как исследуемые в [32] образцы представляли собой многослойные пленки, в то время как программа, основанная на уравнениях (5) и (6) может быть использована только для монокристаллических пленок. Тем не менее, не представляет особых трудностей усовершенствовать применяемые здесь методы и таким образом рассчитать возможные вихреподобные образования в сверхрешетке $\mathrm{Co} / \mathrm{Pt}[32]$. Однако данная задача требует отдельного исследования.

Следует отметить, что в работе [32] был также проведен численный анализ на основе открытого программного кода микромагнитного моделирования ОOMMF [34] и были получены возможные микромагнитные структуры, возникающие на дефектах в сверхрешетке $\mathrm{Co} / \mathrm{Pt}$. Они соответствуют вихреподобным неоднородностям различной топологии (цилиндрические магнитные домены, магнитные вихри с полярностью кора $m_{z}(0)=-1$ и $m_{z}(0)=1$ и др.), что находится в удовлетворительном согласии с экспериментальными данными [32].

\section{6. Заключение}

В заключении отметим, что особенностью исследуемых неоднородностей является возможность их существования в различных материалах, в которых имеется одноосная анизотропия. Такими материалами могут быть эпитаксиально выращенные пленки ферритов-гранатов и ферритов шпинелей, пленки гексаферритов [6], сплавы вида GdFeCo [35] и т.д., в том числе и многослойные пленки типа $\mathrm{Co} / \mathrm{Pt}$ [34]. Для некоторых из них (например, для пленок ферритов-гранатов) существует развитая технология их синтезирования, причем с требуемыми значениями материальных параметров [6]. Последнее позволяет получить пленки такого состава, в которых могут существовать вихреподобные образования с необходимыми свойствами.

Кроме того, данные неоднородности, могут возникать в пленках, толщина которых может меняться в достаточно широком диапазоне величин. Это объясняется тем, что единственным параметром, определяющим размеры пленок и, соответственно, размеры вихреподобных образований на дефектах является ширина ДГ $\Delta_{1}$, которая для некоторых материалов может составлять величину $\Delta_{1} \sim(50-100) \mathrm{nm}$ [36]. В то же время толщина пленок не может быть настолько малой величиной, при которой $D \ll \Delta_{1}$. В этом случае рассматриваемая модель из-за усиливающегося влияния размагничивающих полей уже не описывает вихреподобные образования с блоховским распределением вектора $\mathbf{m}$. Тем не менее, если провести дополнительный анализ и учесть неелевский вклад в структуру данных неоднородностей, то можно значительно расширить границы применимости расчетов, т.е. использовать их и для ультратонких пленок. Однако рассмотрение такой задачи выходит за рамки данной работы.

\section{Список литературы}

[1] Р.М. Вахитов, Е.Б. Магадеев. ФММ 115, 306 (2014).

[2] D. Gall. In: Handook of Magnetism and Advanced Magnetic Materials. V. 2. Micromagnetism / Eds H. Kronmuller, S. Parkin. John Willey \& Senc. Ltd. N. Y. (2007). P. 1023.

[3] В.В. Федотова, А.П. Гесь, Т.А. Горбачевская. ФТТ 37, 2835 (1995).

[4] В.К. Власко-Власов, Л.И. Дедух, М.В. Инденбом, В.К. Никитенко. ЖЭТФ 84, 277 (1983).

[5] Р.М. Вахитов, Т.Б. Шапаева, Р.В. Солонецкий, А.Р. Юмагузин. ФММ 118, 571 (2017).

[6] С. Тикадзуми. Физика ферромагнетизма. Магнитные характеристики и их практические применения. Мир, М. (1987). 419 c.

[7] Р.М. Вахитов, Е.Б. Магадеев, А.Р. Юмагузин, Р.В. Солонецкий. ФТТ 57, 1462 (2015).

[8] R.M. Vakhitov, R.V. Solonetsky, A.A. Akhmetova. Book of Abstracts. Moscow International Simposium on Magnetism (1-5 July, 2017, Moscow). PrintLETO. (2017). P. 380.

[9] Ryan V. Mityushev, V.M. Vinokur, L. Berlyand. Sci. Rep. 5, 7821 (2015).

[10] А.А. Белавин, А.М. Поляков. Письма в ЖЭТФ 22, 245 (1975).

[11] И.Е. Дзялошинский, Б.А. Иванов. Письма в ЖЭТФ 29, 592 (1979).

[12] А.С. Ковалев, А.М. Косевич, К.В. Маслов. Письма в ЖЭТФ 30, 321 (1979).

[13] N.A. Usov, S.E. Peshany. JMMM 118, 2290 (1993).

[14] A. Bogdanov, A. Hubert. JMMM 138, 255 (1994).

[15] T. Shinjo, T. Okuno, R. Hassdorf, K. Shigeto, T. Duo. Science 289, 930 (2000).

[16] K.Y. Guslienko, J. Nanosci. Nanotechnol. 8, 2745 (2008).

[17] M. Rahm, J. Biberger, V. Umansky, D.J. Weiss. Appl. Phys. 95, 6708 (2004).

[18] A.R. Pereira. J. Appl. Phys. 97, 094303 (2005). 
[19] F.A. Apolonio, W.A. Moura-Melo, F.P. Crisafuli, A.R. Pereira, R.L. Silva. J. Appl. Phys. 106, 084320 (2009).

[20] D. Toscano, S.A. Leonel, P.Z. Coura, F. Sato, R.A. Dias, B.V. Costa. Appl. Phys. Lett. 101, 252402 (2012).

[21] А.С. Логгинов, А.В. Николаев, Е.П. Николаева, В.Н. Онищук. ЖЭТФ 117, 571 (2000).

[22] K.H. Prabhakara, M.D. Davydova, K.A. Zvezdin and et al. Book of abstracts. PrintLETO, Moscow. (2017). P. 748.

[23] Е.Б. Магадеев, Р.М. Вахитов. ФТТ 53, 944 (2011).

[24] Е.Б. Магадеев, Р.М. Вахитов. ТМФ 171, 511 (2012).

[25] A. Hubert, R. Schafer. Magnetic Domains. Springer, Berlin, Heidelberg, N. Y., 3rd Printing (2009). 707 p.

[26] A. Sakuma. JMMM 88, 369 (1990).

[27] A.H. Bobeck, Della Torre E. Magnetic bubbles. Amsterdam. N. Y., North Holland, (1975).

[28] W.J. DeBonte. J. Appl. Phys. 44, 1793 (1973).

[29] Р.М. Вахитов, Р.В. Солонецкий, И.Б. Ларионов. ФТТ 59, 1089 (2017).

[30] B.A. Lilley. Phil.Mag. 41, 792 (1950).

[31] К.П. Белов, А.К. Звездин, А.М. Кадомцева, Р.З. Левитин. Ориентационные переходы в редкоземельных магнетиках. Наука, М. (1979). 320 с.

[32] M.V. Sapozhnikov, S.N. Vdovichev, O.L. Ermolaeva, N.S. Gusev, A.A. Fraerman, S.A. Gusev, Yu.V. Petrov. Appl. Phys. Lett. 109, 042406 (2016).

[33] T. Devolder, J. Ferre, C. Chappert, H. Bernas, J.-P. Jamet, V. Mathet. Phys. Rev. B 64, 064415 (2001).

[34] M.J. Donahue, D.G. Porter. OOMMF User's Guide Version 1.0, National Institute of Standards and Technology, Gaithersburg, MD (1999).

[35] W. He, H.-L. Liu, H.-Y. Wu, J.-W. Cai, Z.-H. Cheng. Appl. Phys. Lett. 106, 042401 (2015).

[36] А.М. Балбашов, Ф.В. Лисовский, В.К. Раев и др. / Элементы и устройства на цилиндрических магнитных доменах. Справочник / Под ред. Н.Н. Евтихиева, Б.Н. Наумова. Радио и связь, М. (1987). 488 с.

Редактор Т.Н. Василевскал 